\title{
Vorwort
}

\section{Grundzüge der vorliegenden Ausgabe}

1. Wo meine Angaben über Lesarten der Hss. von denen von Gaisford, Heikel und Gifford abweichen, sind meine die richtigen; ich habe nämlich in allen solchen Fällen die Lesarten in den betreffenden Hss. noch einmal überprüft. Und wo z. B. Dindorf eine andere Lesart als mein Text bietet, ohne daß ich im kritischen Apparat etwas bemerke, dort ist meine Lesart die von allen Hss. außer etwa $\mathrm{D}$ gebotene. Daher sind testimonia ex silentio bei meinem Text durchaus zuverlässig (soweit es in menschlichen Dingen überhaupt eine Zuverlässigkeit gibt). Im übrigen habe ich mich bemüht, den kritischen Apparat soviel als möglich positiv zu fassen. Die Zusammenfassung von Hss. (etwa BON) durch Siglen ist m. E. nicht am Platz, da die Versuchung, Siglen auch dort zu setzen, wo die eine oder die andere der auf solche Weise zusammengefaßten Hss. nicht mit den übrigen übereinstimmt, nur allzu nahe liegt. Ich bin der Ansicht, daß jede Hs. für sich Zeugnis ablegen soll.

2. Der Vulgattext des Stephanus und Vigerus hat bei der Herstellung des Textes gänzlich auszuscheiden. Daß sich bisher davon kein Herausgeber (auch Gifford nicht) vollständig frei gemacht hat, dafür drei bezeichnende Beispiele: a) Bd. I S. 583, 13 heißt im Zitat

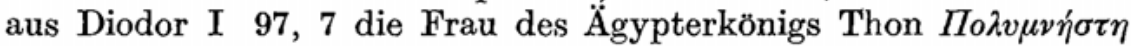
(im Genetiv); aber alle bisherigen Herausgeber der P. E. schreiben

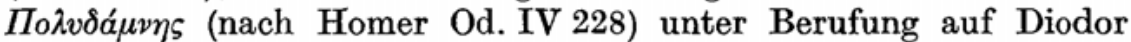
und ebenso alle Herausgeber Diodors unter Berufung auf Eusebius; in Wahrheit aber bieten sämtliche Hss. sowohl des Diodor wie des

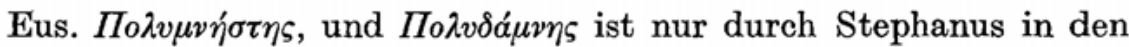
Text gekommen!! S. Mras, Rheinisches Museum N. F. 92 (1944) 218. b) Bd. II S. 240, 18 las man bisher (auch noch bei Gifford) folgen-

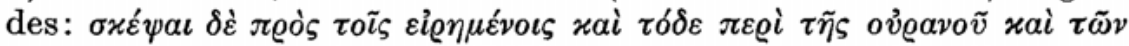

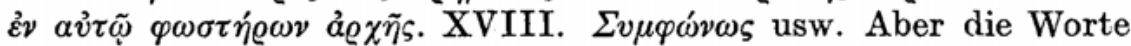
$\pi \varepsilon \varrho i$ ( $\tau \tilde{\eta} \varsigma$ fehlt) ov $\varrho \alpha \nu o \tilde{v}$ bis $\varphi \omega \sigma \tau \eta \dot{\eta} \varrho \omega \nu$ sind nichts anderes als die (in allen Hss. stehende) Überschrift des nächsten (18.) Kapitels, sie sind aber in $\mathbf{I}$ und daher auch in $\mathbf{E}$ (einer der beiden Grundlagen der Aus- 
gabe des Stephanus), weil mit schwarzer Tinte geschrieben, vom

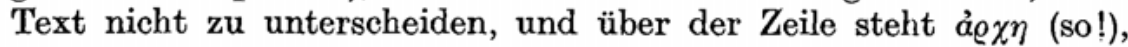
d. h. der Schreiber markierte damit den Anfang des 18. Kap. bei $\Sigma v \mu$ -

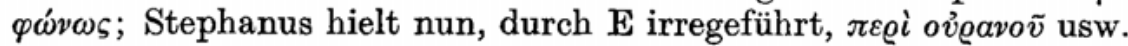

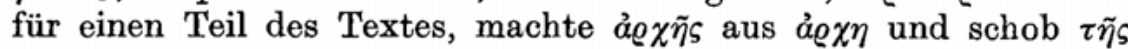
nach $\pi \varepsilon \varrho i$ ein, und das haben ihm alle bisherigen Herausgeber nachgeschrieben! S. Mras a. a. O. 224f. c) Bd. II S. $425,11 \mathrm{ff}$.

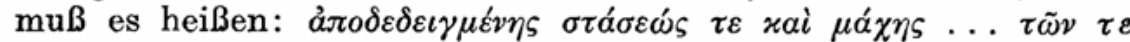
$\lambda o เ \pi \tilde{\omega} v$ á $\pi \dot{\alpha} v \tau \omega \nu \ldots d \dot{\alpha} \tau \varepsilon \lambda \eta \lambda \varepsilon \gamma \mu \varepsilon \dot{v} \omega \nu$ : $\tau \varepsilon$ nach $\tau \tilde{\omega} \nu$ ist unentbehrlich (es verbindet ja ả $\pi \varepsilon \lambda \eta \lambda \varepsilon \gamma \mu \varepsilon \dot{v} \omega \nu$ mit $\alpha \jmath \pi o \delta \varepsilon \delta \varepsilon \imath \gamma \mu \varepsilon \dot{v} \eta \varsigma$ ), steht auch tatsächlich in allen Hss. außer in D, fehlt daher bei Stephanus und allen übrigen Herausgebern bis auf Gifford einschließlich.

3. Nicht bloß die den Büchern vorangeschickten Inhaltsangaben, sondern auch die den Kapiteln in den Hss. vorangehenden Überschriften hat Eus. für sein Werk bestimmt. Mit Recht hat J. Bidez in seiner Besprechung der Giffordschen Ausgabe (Revie critique N. S. 61 [1906] 508) darüber geklagt, daß die Herausgeber von Gaisford an diese Überschriften weggelassen haben. Daß sie von Eusebius herrühren, dafür gibt es schlagende Beweise:daß das dritte Kapitel des vierten Buches aus einem Werk des Diogenianus stammt, erfahren wir weder im Text noch im Inhaltsverzeichnis des vierten Buches, sondern nur aus der Kapitelüberschrift (Bd. I S. 169, 21); der Titel der Schrift des Porphyrius sowie die Nummer des Buches, dem das dritte Kapitel des zehnten Buches entnommen ist, wird bloß in der Kapitelüberschrift angegeben (Bd. I S. 561, 12f.); Buch XI Kap. 30

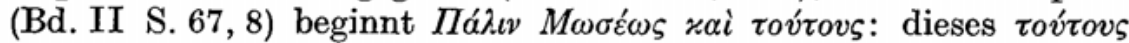
ist unverständlich ohne die vorhergehende Kapitelüberschrift $\Pi \varepsilon \varrho i$

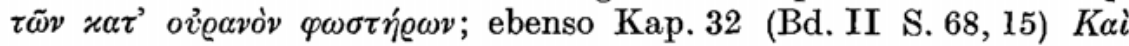

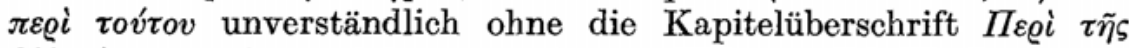

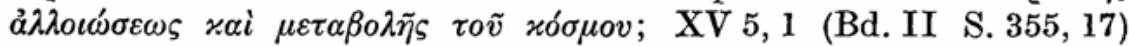
$\pi \varrho o ̀ \varsigma ~ \tau o \tilde{v} \delta \eta \lambda \omega \vartheta \varepsilon ́ v \tau o \varsigma:$ wer das ist, ergibt sich nur aus der Kapitelüberschrift. Der zitierte Autor und sein Werk sind nur in Überschriften zu Kapiteln oder Abschnitten in folgenden Fällen angegeben: IX 14, 3 (Bd. I S. 500, 9f.); X 10 (Bd. I S. 591, 6): erst

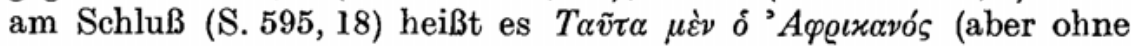
Angabe des Werkes; es fehlt auch in der Inhaltsangabe des Buches); XIV 17 (Bd. II S. 303, 11f.): in der Inhaltsübersicht des Buches ist bloß der Autor angegeben ${ }^{1}$ ); XIV 22 (Bd. II S. 320, 13): Angabe auch der Schrift, des Philebos (im Inhaltsverzeichnis des Buches

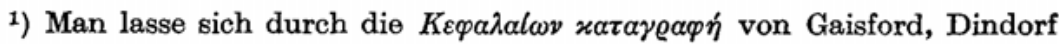
und Gifford nicht täuschen: sie bieten ein Gemengsel aus den Inhaltsangaben der Bücher und den Kapitelüberschriften. 


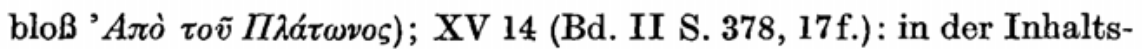
angabe des Buches fehlt Autor und Werk; ebenso XV 17 (Bd. II S. 381, 9). Wie man sieht, sind die genaueren Angaben in den Kapitelüberschriften, was ja auch ganz natürlich ist: der Autor gibt zunächst zu jedem Kapitel die entsprechende Inhaltsangabe; Sache der Abschreiber ist es dann, diese Kapitelüberschriften am Anfang jedes Buches zusammenzustellen. So erklären sich manche kleine Abweichungen (die jedoch nie Widersprüche sind). Selbstverständlich hat Eusebius auch diese Zusammenstellungen vor jedem Buch angeordnet.

4. Was die Herstellung des Textes betrifft, so folge ich in den Büchern VI bis XV dort, wo I ausführlicher als die andere Klasse ist, dieser Hs., wo das aber nicht der Fall ist, treffe ich die Entscheidung zwischen beiden Klassen je nach der Sachlage; im allgemeinen kann man sagen, $\mathrm{da} B$ in den ersten fünf Büchern dort, wo A und I übereinstimmen, die von ihnen gebotene Lesart meist die richtige ist; dasselbe gilt in den Büchern VI bis XV von der Übereinstimmung der Hss. B und I. Eusebius ist als Stilist kein Purist. So sehr er gedanklich und sachlich von Clemens abhängig ist, in seinem Stil ist er von diesem (der mit der zweiten Sophistik kokettiert) ganz unbeeinflußt. Seine Diktion zeigt vielmehr Beziehungen zum Homilienstil des Origenes. Dazu kommen aber noch Weitschweifigkeit und Rechenfreudigkeit (in chronologischen Dingen), Eigenschaften, die ihn als Orientalen charakterisieren. Das Verkennen des Stilcharakters des Eusebius und der von ihm zitierten Autoren hat manche Gelehrte zu überflüssigen Konjekturen verleitet (was hat z. B. Kuiper am Ezechiel herumgedoktert!). Ich habe mich des gewaltsamen Eingreifens in den Text soweit als möglich enthalten, konnten doch an vielen Stellen mit nur geringen Änderungen

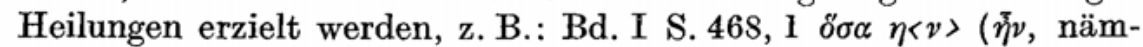
lich $\lambda \alpha \beta \varepsilon \tilde{\imath} v)$; Bd. I S. 477, $16 \mathrm{f}$. $\chi \omega \dot{\omega} \varrho \alpha \tilde{\eta} K v \kappa \lambda \dot{\omega} \pi \omega \nu$ statt $\chi \dot{\omega} \varrho \alpha \delta^{\prime} \dot{\eta}$ $K$. (also $\delta$ falsch für $\tau$ ); Bd. I S. 478, 7 ä̈̊iov (gemeint ist die ewig feuchtwarme Atmosphäre, der Indien seine üppige tropische Vegetation verdankt) statt aĩiov (also umgekehrt fälschlich $\tau$ für $\delta$ ); Bd. I S. 503, $4\langle\hat{\eta}\rangle \dot{\varepsilon} v$; Bd. I S. 506, 3 hat die (bloß optische) Änderung von $\mu v \varrho i o v$ in $\mu \eta \varrho i o v$ und die Erklärung von $\varepsilon^{\prime} x \lambda v o v$ als हैं $x-\lambda v o v$ $(=\hat{\varepsilon} \xi \xi \hat{\varepsilon} \lambda v o v)$ die sieben als ganz unverständlich verschrienen Verse

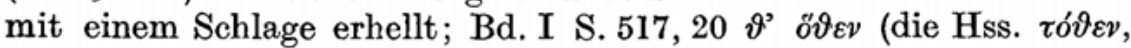

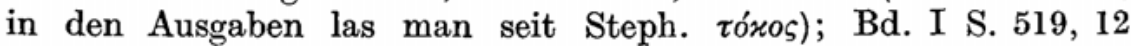

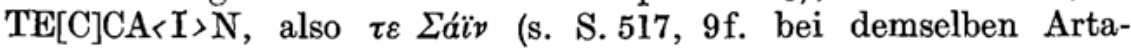

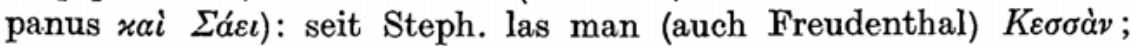

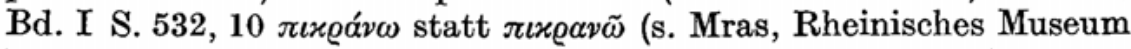
N. F. 92 [1944] 229); öfter war $\gamma \varepsilon$ für $\tau \varepsilon$ zu setzen, so Bd. II S. 23, 3 
( $\tilde{\tau} \iota \gamma \varepsilon)$, und umgekehrt $\tau \varepsilon$ für $\gamma \varepsilon$ wie Bd. II S. 167, 7 ( $\tau \tilde{\omega} \nu \tau \varepsilon \varepsilon \dot{\varepsilon} \kappa:$ zwei Glieder: 1. $\pi \circ \imath \eta \tilde{\omega} v-\vartheta \varepsilon o \lambda{ }^{\gamma} \omega v$, 2. $\tau \tilde{\omega} v-\vartheta \varepsilon \tilde{\omega} v$ ); Bd. II S. 228, 18 $\eta \tilde{~(d i e ~ H s s . ~} \hat{\eta})$ : die heiligen Schriften der Hebräer haben (im Gegensatz

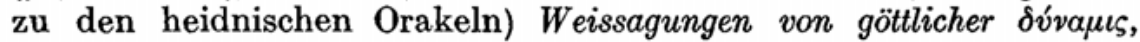
soweit dies menschlichem Fassungsvermögen entspricht. Manche Stellen waren überhaupt nicht zu ändern, sondern nur richtig zu erklären,

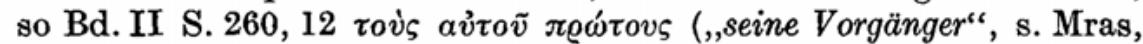

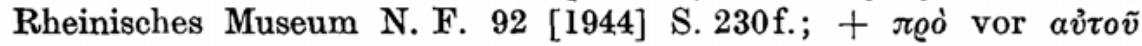
Steph., seitdem in allen Ausgaben).

5. Bezüglich derjenigen von Eusebius zitierten Autoren, die uns durch ihre eigenen Kodizes erhalten sind, bemerke ich, daß ich die von Eusebius angeführten Stellen so biete, wie sie dieser verstanden (oder auch mißverstanden) hat, und in der Form, in der er sie uns darbieten wollte. Es wäre verkehrt, den Text des Eus. nach einem solchen Autor ändern zu wollen ${ }^{1}$ ); das gilt insbesondere von den zahlreichen Platostellen, deren nicht wenige er anders als wir verstanden (oder mißverstanden) hat (nur wo aus den Hss. des Eus. gar kein Sinn hervorgeht und sie atgenscheinlich verderbt sind, bin ich zu Änderungen an der Hand des betreffenden Autors geschritten). So ist Bd. I S. 25, 16 (aus Diodor I 6,3) éxeivoıs gewiß falsch (es müßte $\dot{\varepsilon} x \varepsilon i v \omega$, nämlich $\tau \tilde{\omega}$ xó $\sigma \mu \omega$ heißen, wie man in den modernen Ausgaben Diodors tatsächlich liest), aber da auch die Hss. Diodors Éxzivoıs haben, folgt daraus, daß so bereits Euseb. in seiner Diodorausgabe las, es darf also hier bei ihm nichts geändert werden ${ }^{2}$ ).

6. Auf die Interpunktion habe ich großen Wert gelegt und durch Änderung derselben nicht wenige Stellen geheilt. Das bisher übliche Überma $B$ an Beistrichen habe ich, um die Unterbrechung des gedanklichen Zusammenhanges zu vermeiden, möglichst eingeschränkt.

7. Da die P. E. keine leichte Lektüre ist und die Kenntnis des Griechischen bei der jüngeren Generation nicht mehr sehr tief geht und voraussichtlich immer geringer werden wird, habe ich Erklärungen und Übersetzungshilfen gelegentlich eingestreut, bei denen ich dem Umstand Rechnung zu tragen suchte, daß die P. E. ja nicht bloß für Philologen, sondern auch für Historiker, Philosophen, Theologen und andere Gelehrte wichtig ist.

8. Sowohl das Stellen- wie das Namen- und Sachregister habe ich von Grund auf neu angelegt und auf die Anfertigung des letzteren

1) Diesen Grundsatz hat bereits Dindorf I S. XVIIf. angedeutet, aber selber nur selten befolgt.

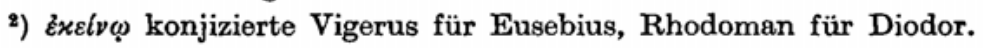


große Mühe verwendet ${ }^{1}$ ), um den Inhaltsreichtum und die Gedankenfülle der P. E. der Öffentlichkeit zugänglich zu machen.

9. Neu ist auch das Sprachregister. Von den bisherigen Herausgebern bieten bloß Heinichen (II 462-476) einen Index verborum et formularum loquendi und Gifford (IV 555-575, ohne Erläuterungen) einen Index of Greek Words. Über die Grundsätze, nach denen ich das Sprachregister ausgearbeitet habe, handle ich in der Einleitung zu diesem. Jedenfalls geht es bei einem Autor, von dem kein vollständiges Wörterverzeichnis angelegt wird, nicht an, gewöhnliche Wörter in gewöhnlichen Bedeutungen zu buchen (wie z. B. rv́vaıov,

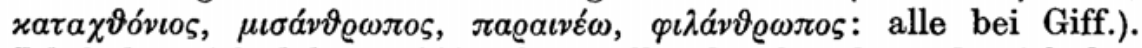
Ich habe mich dabei auf Eusebius selber beschränkt und mich bemüht, seine schriftstellerische Persönlichkeit herauszuarbeiten (dieser Absicht dient auch der Teil III 3: Stilistik). Der Abschnitt III 2 soll Bausteine für die Grammatik späterer griechischer Autoren liefern. Gern hätte ich das Sprachregister auf die nur durch Eus. erhaltenen Schriften ausgedehnt, doch haben leider die Kriegsverhältnisse meine Absicht verhindert. Der Anhang enthält alle Scholien.

Am Schlusse meiner Arbeit blicke ich auf mehr als vierzig Jahre zurück. Ende 1913 ersuchte mich mein Lehrer Hans von Arnim, diese Ausgabe zu übernehmen. 1914 lie $\beta$ ich auf diplomatischem Wege mein Gesuch um Übersendung der Hs. B an die hiesige Hofbibliothek der Pariser Nationalbibliothek überreichen. Allein bevor das Gesuch erledigt wurde, brach der erste Weltkrieg aus. Erst neun Jahre später konnte ich daran denken, das Ansuchen zu wiederholen, diesmal mit Erfolg; im Dezember 1923 traf der Kodex B aus Paris in Wien ein. Nach der Kollationierung dieser Hs. unternahm ich in den folgenden Jahren wiederholt Reisen, zum Teil mit Unterstützung der damaligen Preußischen Akademie der Wissenschaften, nach Frankreich und Italien, um die übrigen Hss. zu vergleichen. Meine Ernennung zum Ordinarius für klassische Philologie an der Wiener Universität brachte schwere Aufgaben mit sich (700 und mehr Hörer gab es in jedem Semester!), Anforderungen, die den Fortgang meiner Ausgabe zunächst hemmten. Neue Hemmnisse brachte der zweite Weltkrieg. Wegen zunehmender Bombenangriffe mußte ich im Juli 1944 mein Ms. verlagern. Nachdem ich es am 15. Jänner 1947 unversehrt zurückbekommen hatte, wurde davon an der photographischen Anstalt der hiesigen Nationalbibliothek seit Juli 1949 ein Kleinfilm hergestellt. Am 13. November 1950 erhielt ich die ersten Korrekturbogen. Der Druck wurde aber einige Male durch monatelange Pausen unterbrochen. Für ihre Beteiligung

1) Noch Gifford bietet den Index rerum et nominum des Vigerus. 
an der ersten Korrektur danke ich besonders den Herren Professoren D. Dr. Otto EiBfeldt (bis zum 7. Buch) und Dr. Max Rauer. Zu außerordentlichem Dank aber fühle ich mich Herrn Dipl.-Phil. Eberhard Rechenberg verpflichtet für die sorgfältige und gewissenhafte Überwachung des Druckes.

Und so habe ich endlich nach mancherlei Hindernissen diese Ausgabe, die ich als junger Mann übernahm, als Greis glücklich vollendet.

Wien.

Dr. Karl Mras, ord. Universitätsprofessor und wirkliches Mitglied der Österreichischen Akademie der Wissenschaften. 